\title{
Research on the Prediction of the E-commerce Profit Based on the Improved Parallel PSO-LSSVM Algorithm in Cloud Computing Environment
}

\author{
Zhihui Fu \\ Department of Information Technology, Zhejiang Business College, Hangzhou, \\ China \\ 511710938@qq.com
}

\begin{abstract}
E-commerce is a cyber, electronic and informational business activities. Electronic commerce has been developing rapidly in recent years in China. Cloud computing provides a new idea for the development of electronic commerce. In practice, cloud computing also has a very good combination of e-commerce. To predict the profit of electronic commerce can find the existing problems, grasp the development trend, and make better management of electronic commerce. In this paper, we study the profit prediction of electronic commerce. Then, we propose an improved parallel PSO-LSSVM algorithm, and use this algorithm to predict the benefits of electronic commerce. Experimental results show that the proposed algorithm is effective and reliable.
\end{abstract}

Keywords: E-commerce, Cloud computing, Prediction

\section{Introduction}

The continuous development of the Internet has prompted the emergence of the cloud computing technology [1]. The cloud computing not only completely changes the business model and the way that people work, but also brings the development rapidly of the e-commerce industry to a new opportunity [2-3]. The combination between the cloud computing and the e-commerce has risen the e-commerce to a new height. The development of the electronic commerce needs a good management. The profit prediction of the e-commerce is one aspect of the good management. The e-commerce and the cloud computing can reach the "win-win".

At present, many scholars have conducted a research on the e-commerce. Wang Yi researched on the customer behavior and the sales forecasting model in the electronic commerce [1]. The author thought that the website's visit and the purchase transaction behavior could produce the massive data in the electronic commerce environment. These data contained a large number of potentially useful information for the market analysis and prediction. Depth analysis of the information not only provided the basis of the decision making for the enterprises to improve the electronic commerce website quality, but also had the important practical significance to improve the operating efficiency of the electronic commerce. Based on these data, the author made research on the problems of the electronic commerce, such as the customer's visit behavior, the sales forecast and so on. According to the shortcomings of the current electronic commerce customer churn prediction model, Ren Jianfeng and Zhang Xinxiang proposed an e-commerce customer churn prediction model which was combined with the genetic algorithm, support vector machine and BP neural network [5]. The simulation results showed that the combined model could improve the accuracy of the customer churn prediction and speed up the prediction speed compared with the single prediction model. Zhang Yanrong proposed an improved attribute reduction algorithm based on discernibility matrix. In addition, the 
author got an e-commerce consumer behavior prediction method based on rough sets by extracting and reducing the generated rules [6]. In order to improve the accuracy of the customer churn prediction at the individual level, Ju Chunhua, Lu Qibei and Guo Pengfei set up an electronic commerce customer churn prediction model which was integrated into the individual activity [7]. Xia Han structured the enterprise electronic commerce credit risk predictability index based on enterprise accounting index and electronic commerce operation ability. Then, the author forecasted the credit risk degree of electronic commerce through the PCA and LSSVM [8]. Zi Zhidao simulated and predicted the development of the cross-border e-commerce in China with the grey prediction model based on 2008 to 2012 cross-border e-commerce business data. Then, the author forecasted cross-border e-commerce trade volume value from 2013-2015. At last, the author pointed out that Chinese cross-border e-commerce would be greater development after the reform and innovation of the leading policy [9]. In addition, there are many scholars having conducted a study of electronic commerce [10-13].

Eleni Kavvadia etc. introduced a novel cloud computing network architecture that allowed for the formulation of the optimization as an Uncapacitated Facility Location (UFL) problem, where a facility corresponded to an instantiation of a particular service (e.g. a virtual machine) [14]. Then, a policy that employed virtual machine replication and merging along with migration was proposed to reduce the overall cost for using a service. Omar Ali, Jeffrey Soar and Jianming Yong indicated that the factors in the extant literature were supported; additional challenges and issues emerged which were related to effective network, data storage location, availability of different service providers, policy makers, a limited understanding of the cloud and business transformation [15]. Alireza Shameli-Sendi etc. believed that cloud computing technology could substantially change the way we responded to a DDoS attack, based on a number of new characteristics, which were introduced with the advent of this technology [16]. Their objective was to show how these characteristics bring a novel perspective to existing DDoS mechanisms, and so gave researchers new insights into how to mitigate DDoS attacks in the cloud computing. Ibrahim Alzamil etc. proposed a system architecture that could be used for profiling the resources usage in terms of the energy consumption [17]. The results of the conducted experiments showed energy-awareness at physical host and virtual machine levels. Youming Lin etc. addressed remote display access as a means for mobile cloud computing, with focus on its power consumption at mobile devices [18]. In addition, many scholars have carried out a study on cloud computing [19-23].

In this paper, we combine the electronic commerce and the cloud computing and propose the improved parallel PSO-LSSVM algorithm. In the experiment, we predict the profit of the e-commerce. The structure of this paper is as follows. The first part is the introduction. In this part, we mainly introduce the related background. The second part is the cloud computing. In this part, we introduce the concept of cloud computing. The third part is the improved parallel algorithm. In the part 3, we combine the PSO and LSSVM algorithm and propose an improved parallel PSO-LSSVM algorithm. The fourth part is the experiment and the fifth part is conclusion.

\section{Cloud Computing}

Cloud computing is a comprehensive development of the distributed computing, parallel computing and grid computing. The cloud computing is the result of the application of conceptual combination of Utility Computing, Virtualization, HaaS, SaaS, PaaS. Cloud computing refers to an application that the network provide by serve. It also refers to hardware and system software that is used to provide these services in a data center. The concept of cloud computing is developed on the basis of SaaS (software as a 
service, software as a service). The following figure shows the cloud computing users and providers in different levels of cloud computing.

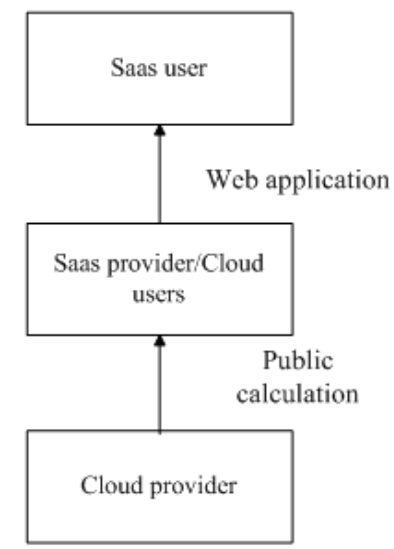

Figure 1. Cloud Computing Users and Providers

Hadoop is an open source framework for Apache open source organization, which has been applied to many large web sites. The advantage of Hadoop is as follows.

The first is the extended: the expansion of storage and calculation is the design basement of Hadoop. The Hadoop extension is very simple and it does not need to modify any existing structure.

The second is the Economy: the framework can be run on any ordinary PC and there are no special requirements on the hardware.

The third is the Reliability: the backup and recovery mechanism of the distributed file system and the task monitoring of MapReduce guarantee the reliability of the distributed processing. The Hadoop provides more than 1 backup by default, and the last is the efficient. The Efficient data exchanging in a distributed file system and MapReduce combining with local data processing mode are prepared for high efficient processing of massive information

Hadoop distributed file system (HDFS) is designed to be suitable for running on the common hardware (hardware commodity) of the distributed file system. HDFS is a highly fault-tolerant system that is suitable for deployment on cheap machines. HDFS can provide high throughput data access and is very suitable for the application of big data sets. HDFS relaxes a part of the POSIX constraints to achieve the purpose of streaming data to read the file system. HDFS is developed as a basis for the Nutch Apache search engine. Then, the HDFS developed independently as part of the Hadoop Core Apache project.

MapReduce is actually the process of divide/conquer. A Map/Reduce job usually separates the input data sets into several independent data blocks, which are processed by the map task in a completely parallel manner. The framework will sort the output of the map first, and then input the results to the reduced task. Usually, the input and output of the job will be stored in the file system. The whole framework is responsible for scheduling and monitoring tasks, as well as the re-implementation of the failed tasks.

Fig. 2 shows the architecture of the Hadoop distributed system. As is shown in the figure, they are generally composed of a master and workers. Master is responsible for workers maintaining, the establishment of user query execution plan, co-ordinate the whole process of query execution. The master is responsible for handling user queries according to the query plan. 


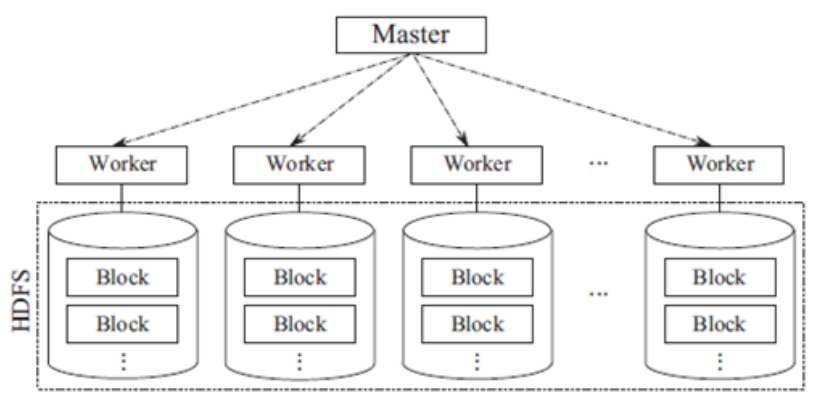

Figure 2. Architecture of Hadoop-based Distributed Systems

\section{The Improved Parallel Algorithm}

\subsection{PSO}

Swarm Optimization Particle (PSO) is a global search optimization algorithm proposed by the American scholar Kennedy and Eberhart. Based on swarm intelligence theory, PSO algorithm is used to operate the position and velocity of the particles in the population, which can achieve the goal of competition and cooperation among the particles. Compared with the traditional evolutionary algorithms, the PSO algorithm avoids the complexity of genetic operation. At the same time, the PSO keep the population-based global search optimization strategy. The unique memory ability of PSO can adjust the search direction and speed according to the current individual and the overall situation. Each generation of particles in the population has the ability to learn and self-learning, so the PSO algorithm can search the global optimal position of the population in less iteration times. At present, the PSO algorithm has been widely used in the fields of function optimization, data mining, artificial neural network training and so on.

Particle swarm optimization algorithm achieves the updating of the position and velocity through the competition and cooperation of the particles in population. These can achieve the goal of searching for the global optimal solution in the complex space. The PSO has the characteristics of swarm intelligence and evolutionary computation.

In the PSO algorithm, a particle in the population is a solution of the space of the optimization problem. Algorithm starts by initializing particle, namely initializes $m$ random particles and the algorithm constitute them a population $X=\left\{X_{1}, X_{2}, \cdots, X_{m}\right\}$. Then, the positions of each particle $X_{i}=\left\{X_{i 1}, X_{i 2}, \cdots, X_{i m}\right\}$ is a solution for the optimization problem. The fitness value of each particle is determined by the objective function. Each particle will search iteratively to adjust their positions to find new solutions in the solution space through update speed $V_{i}=\left\{V_{i 1}, V_{i 2}, \cdots, V_{i m}\right\}$ constantly. In the constant iteration, the particle will adjust itself according to the global extremum and the individual extremum. Individual extremum $P_{i d}$ is the best position of the particle itself. Global extremum $P_{g d}$ is the optimal position of the whole population. When the two extremes are found, each particle adjusts its velocity and position iteratively according to the formula 1 and 2 .

$$
\begin{gathered}
v_{i d}^{k+1}=w v_{i d}^{k}+c_{1} r_{1}\left(p_{i d}^{k}-x_{i d}^{k}\right)++c_{2} r_{2}\left(p_{g d}^{k}-x_{i d}^{k}\right) \\
x_{i d}^{k+1}=v_{i d}^{k+1}+x_{i d}^{k}
\end{gathered}
$$

The $v_{i d}^{k+1}$ is the velocity of the $i$ particle in the $d$ dimension at the $k+1$ iteration. The $x_{i d}^{k+1}$ is the position of the $i$ particle in the $d$ dimension at the $k+1$ iteration. $w$ is the inertia weight. $c_{1}$ and $c_{2}$ are acceleration constant. $r_{1}$ and $r_{2}$ are the random 
numbers from 0 to 1 . In addition, in order to make the particle speed is not too large, we can set the upper limit of the speed.

From the speed of PSO algorithm update formula (1), we can see that the moving speed and the direction of the particles in the particle swarm is composed of three parts decision. The first part is their original speed $v_{i d}$. The second part is the distance between the particle and the best position of the population $p_{g d}-x_{i d}^{k}$. The third part is the distance between the particle and the best position $p_{i d}-x_{i d}^{k}$. They are determined by the weight coefficient, the acceleration constant and their relative importance. When the algorithm can find the global optimal position or the maximum number of iterations, the algorithm reaches the end.

\subsection{LSSVM}

For the second class of linearly separable problems, plane segmentation is able to separate the two types of samples correctly. This can ensure the empirical risk minimum. There are infinite numbers of such planes. But only split plane $\mathrm{H}$ get the longest distance from two kinds of samples. Split plane $\mathrm{H}$ is the optimal classification surface. The optimal classification has the least confidence risk. The principle is shown in the figure 3 .

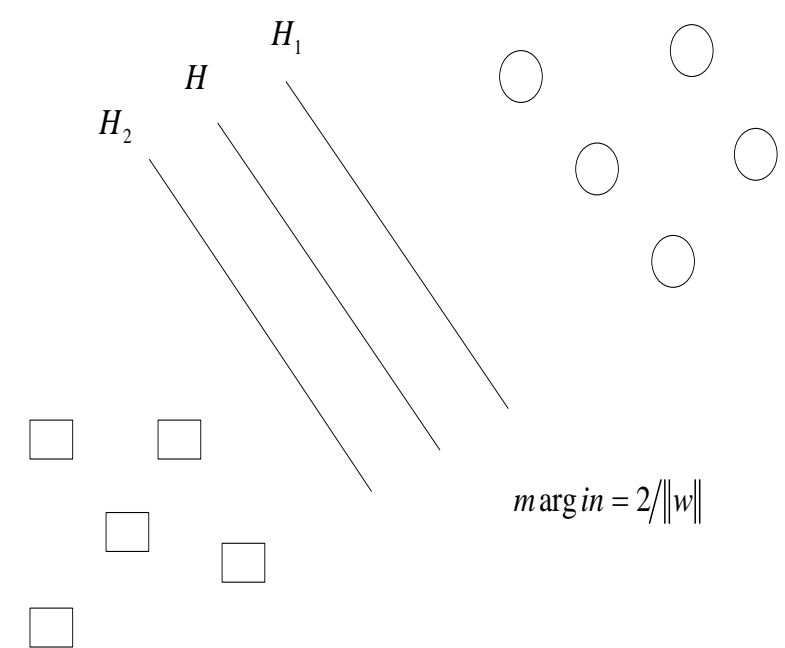

Figure 3. Principle of SVM

Fig.4 is the structure diagram of SVM. By the SVM method, the inner product operation in high-dimensional feature space is replaced by kernel function. When the classification function is constructed, it is not directly to make nonlinear mapping to the sample of the data space. It firstly compares the vectors in data space. Secondly, the results are nonlinear mapping. In this way, a lot of works are carried out in the low dimensional data space, but not in the high dimensional feature space. 


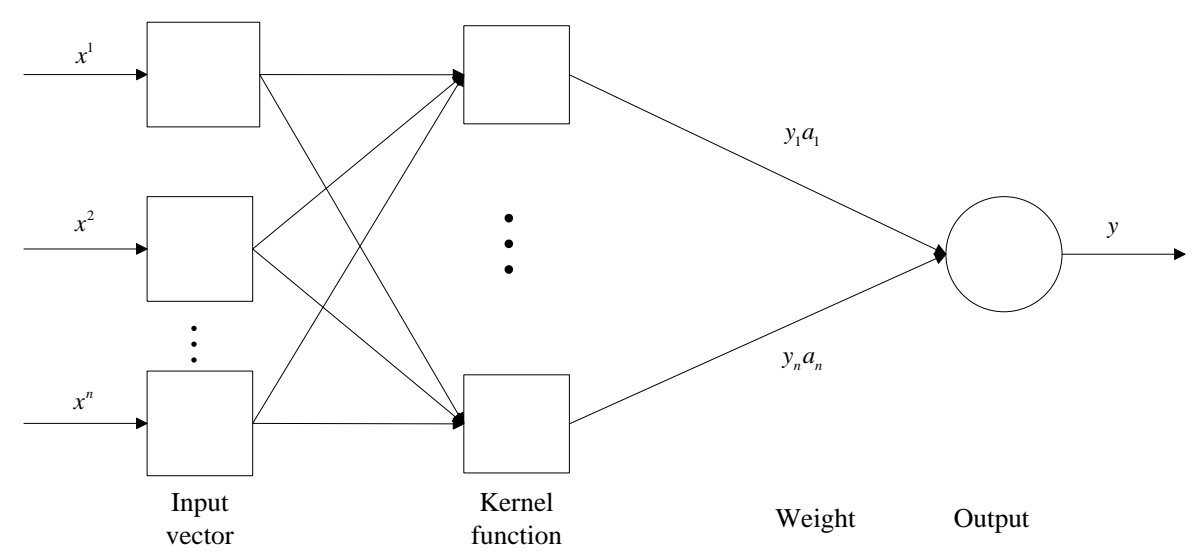

Figure 4. Structure Diagram of SVM

From machine learning loss function, Least Squares Support Vector Machines (LSSVM) uses the two norms in the objective function of the optimization problem, and uses the equality constraints to instead of the inequality constraints in the SVM standard algorithm. These make the solution of the optimal problem of the LS-SVM method become searching for the solution of the linear equations by Kuhn-Tucker condition.

LS-SVM method is to use the equality constraints instead of inequality constraints. The solution process becomes the solution of a set of equations. It avoids the time consuming to solve the two programming problem. So, the solution speed is accelerated. In addition, LS-SVM no longer needs to specify the approximation accuracy and enhances the practicality of the standard SVM model.

The basic method of LS-SVM is as follows.

We define $x \in R^{n}$ and $y \in R$, let $R^{n}$ be the input space, by nonlinear transformation $\phi(\cdot)$, we let in the input space $x$ map into a high dimensional characteristic space.

The linear function of the feature space is defined as,

$$
y=f(x, \omega)=\omega^{T} \phi(x)+b
$$

Where $\omega$ is the weight and $b$ is the skewness.

The objective function is shown as follows.

$$
\min _{\omega, b, \xi} J(\omega, b, \xi)=\frac{1}{2} \omega^{T} \omega+\frac{1}{2} C \sum_{i=1}^{N} \xi_{i}^{2}
$$

s.t.

$$
y_{i}=\phi\left(x_{i}\right) \omega+b+\xi_{i} i=1, \cdots, N
$$

Where $\omega \in R^{h}$ is the weight vector and $\phi(\cdot)$ is non-linear mapping function, $\xi_{i} \in R^{N \times 1}$ is relaxation factor, $b \in R$ is the skewness while $C>0$ is penalty factor.

Importing factors, $\alpha_{i} \in R^{N \times 1}$, we can easily get the function as:

$$
L\left(\omega, b, \xi_{i}, \alpha_{i}\right)=\frac{1}{2}\|\omega\|^{2}+\frac{1}{2} C \sum_{i=1}^{N} \xi_{i}^{2}-\sum_{i=1}^{N} \alpha_{i}\left[\phi\left(x_{i}\right) \omega+b+\xi_{i}-y_{i}\right]
$$

We get 


$$
\begin{aligned}
& \left\{\begin{array}{c}
\frac{\partial L}{\partial \omega}=\omega-\sum_{i=1}^{N} \alpha_{i} \phi\left(x_{i}\right)=0 \\
\frac{\partial L}{\partial b}=\sum_{i=1}^{N} \alpha_{i}=0 \\
\frac{\partial L}{\partial \xi_{i}}=\alpha_{i}-C \xi_{i}=0 \\
\frac{\partial L}{\partial \alpha_{i}}=\phi\left(x_{i}\right)+b+\xi_{i}-y_{i}=0
\end{array}\right. \\
& {\left[\begin{array}{cc}
0 & E^{T} \\
E & \phi \phi^{T}+C^{-1} I
\end{array}\right]\left[\begin{array}{l}
b \\
\alpha
\end{array}\right]=\left[\begin{array}{l}
0 \\
y
\end{array}\right]}
\end{aligned}
$$

Where, $E$ is the matrix that all of the elements are $1 . I$ is the unit matrix with $N \times N$. We use the kernel function to classify the nonlinear regression equation.

Let $\Omega_{i j}=\phi \phi^{T}$,

We can get

$$
\Omega_{i j}=\phi\left(x_{i}\right)^{T} \phi\left(x_{j}\right)=K\left(x_{i}, x_{j}\right)
$$

So, the LS-SVM regression function model is

$$
f(x)=\sum_{i=1}^{N} \alpha_{i} K\left(x_{i}, x_{j}\right)+b
$$

\subsection{Improved Parallel PSO-LSSVM Algorithm}

According to the LSSVM regression theory, we can know that its main parameters are the kernel function parameter $\sigma$ and the penalty parameter $c$. These two parameters have great influence on the learning and the generalization ability of LSSVM. This paper adopts the improved particle swarm optimization algorithm to optimize these two parameters $\sigma$ and $c$. It reduces the blindness of the subjective experience and improves the prediction accuracy in a certain extent. In the PSO algorithm, the updated formula of the position and the velocity is as follows.

$$
\begin{gathered}
v_{i d}^{k+1}=w v_{i d}^{k}+c_{1} r_{1}\left(p_{i d}^{k}-x_{i d}^{k}\right)++c_{2} r_{2}\left(p_{g d}^{k}-x_{i d}^{k}\right) \\
x_{i d}^{k+1}=v_{i d}^{k+1}+x_{i d}^{k}
\end{gathered}
$$

Where, $c_{1}$ and $c_{2}$ are the random number in $[0,1] . w$ is the inertia weight, and $w$ is also the key parameter in the particle swarm optimization algorithm. It can balance the global search ability and the local search ability of the algorithm. In the initial stage of evolution, we hope that the particles have the better explored ability. With the increase of the number of iterations, we hope that the particles have the good development capabilities in the later stage of evolution. Therefore, during the process of the evolution, we need to adjust the inertia weight dynamically.

$$
w=w_{\max }-\frac{1}{2}\left(w_{\max }+w_{\min }\right) \frac{\text { iter }}{\text { iter }_{\max }}
$$

Where, iter is the number of the iterations. iter $_{\max }$ is the maximum number of iterations. $w_{\max }$ is the maximum value of the inertia weight. $w_{\min }$ is the minimum value of inertia weight. $c_{1}$ and $c_{2}$ are the self-learning factor and the social-learning factors. In this paper, we adopt the dynamic adjustment method to obtain the values of $c_{1}$ and $c_{2}$. The specific steps are as follows.

$$
c_{1}=\left(c_{1 e}-c_{1 s}\right) \frac{\text { iter }}{\text { iter }_{\max }}+c_{1 s}
$$




$$
c_{2}=\left(c_{2 e}-c_{2 s}\right) \frac{\text { iter }}{\text { iter }_{\max }}+c_{2 s}
$$

Where, $c_{1 s}$ and $c_{1 e}$ are the initial value and the final value of $c_{1} . c_{2 s}$ and $c_{2 e}$ are the initial value and the final value of $c_{2}$.

From 3.1, we can know that the LS-SVM regression function model is,

$$
f(x)=\sum_{i=1}^{N} \alpha_{i} K\left(x_{i}, x_{j}\right)+b
$$

Then the objective function is,

$$
\begin{aligned}
& \min f(C, \sigma)=\sum_{i=1}^{m}\left(x_{i}-x_{i}\right)^{2} \\
& C \in\left[C_{\min }, C_{\max }\right] \\
& \sigma \in\left[\sigma_{\min }, \sigma_{\max }\right]
\end{aligned}
$$

Where, $x_{i}$ is the output value and $x_{i}$ is the predictive value. The idea of the LSSVM model parameter optimization is to search a set of parameters $(C, \sigma)$ according to the iterative algorithm, and it makes the objective function (17) minimize. This paper adopts the above improved particle swarm optimization (PSO) algorithm to optimize. It makes a set of parameters series $(C, \sigma)$ in domain of definition as the position vector of the particle in the PSO algorithm. The fitness function of each particle is in function (17).

The MapReduce the PSO-LSSVM process is as follows.

Firstly, we assume that the number of the variables of the optimization problem is $n$. We initialize particle swarm. The initial value that the $i$ variable $x_{i}$ produces is $k_{i}$. Then. we use the random method to produce a series of initial values: $x_{1, i}^{(0)}, x_{2, i}^{(0)}, \cdots, x_{k i, i}^{(0)} \in\left[a_{i}, b_{i}\right], i=1,2, \cdots, n$.

Secondly, we make $x_{j, i}^{(0)}$ map into the interval $[0,1]$.

$$
y_{j, i}^{(0)}=\frac{x_{j, i}^{(0)}-a_{i}}{b_{i}-a_{i}}, i=1,2, \cdots, n ; j=1,2, \cdots, k
$$

Thirdly, it produces the chaotic sequences of this generation. We make $y_{j, i}^{(0)}$ as the initial value. Then we use the logistic function,

$$
y_{n+1}=v y_{n}\left(1-y_{n}\right)
$$

Fourthly, $y_{j, i}^{(0, l)}$ can obtain by the inverse mapping,

$$
x_{j, i}^{(0, l)}=a_{i}+\left(b_{i}-a_{i}\right) y_{j, i}^{(0, l)}, i=1,2, \cdots, n
$$

Fifthly, we calculate the fitness value $f\left(x_{j, i}^{(0, l)}\right)$ of the family members.

Sixthly, according to the fitness value $f\left(x_{j, i}^{(0, l)}\right)$ of each generation, we obtain the local optimal adaptive value $p_{i}^{j}$.

Seventhly, according to the local optimal adaptive value $p_{i}^{j}$, we obtain the global optimal adaptive value $g_{\text {Best }}$. If the current evolutionary algebra is smaller than the maximal evolutionary algebra or the current average fitness value minuses the last generation average adaptation value is greater the allowable, $g_{\text {Best }}$ will be optimized of the chaos.

Eighthly, we continue to optimize, get the corresponding parameters and exit the Circulation. 


\section{Experiment}

In order to verify the effectiveness and the feasibility of the improved parallel chaos prediction method, we carry out the numerical simulation experiments to verify. In this experiment, we use 4 RD640 Think Server servers, and each server is a single core PC. We make each core as a Hadoop node. So, there are 4 nodes. One node is as the NameNode, and others are the DataNotes. We make the profit data of 2000 e-commerce shops as the training set from 2006 to 2012. Then, we make the profit data from 2013 to 2015 as the test set. Firstly, we experiment the speedup of the improved parallel PSOLSSVM algorithm. Among them, the acceleration ratio is defined as follows.

$$
S p=T 1 / T p
$$

Where, $T 1$ expresses the transport efficiency under a single processor. $T p$ expresses the transport efficiency under $p$ processor. The experimental results are as follows.

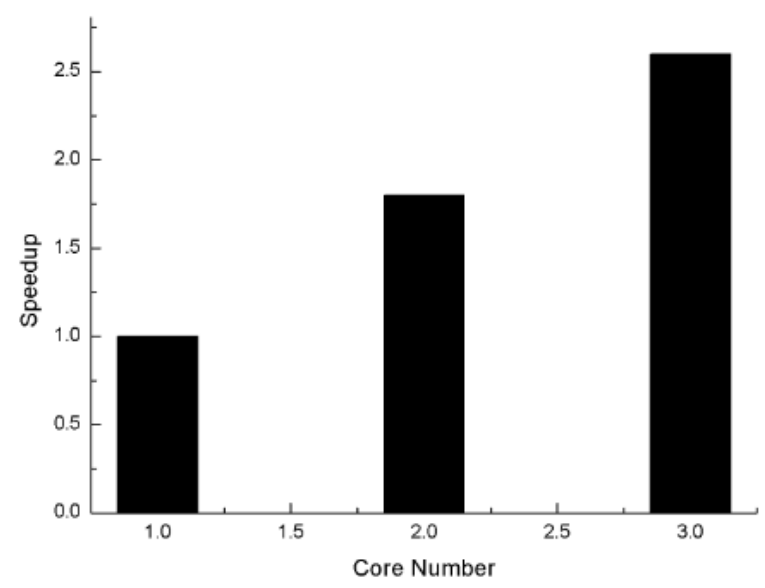

Figure 5. Speedup of the Improved Parallel PSI-LSSVM Algorithm

From the fig 5, we can see that with the increase of the number of processors, the speedup of the improved parallel PSI-LSSVM algorithm is rising. When the number of processors is 3, the speedup is about 2.5 times as much as the number of processors is 1 .

Next, we use the improved parallel PSI-LSSVM algorithm to predict the benefits of the e-commerce shops. Then, the results are as follows.

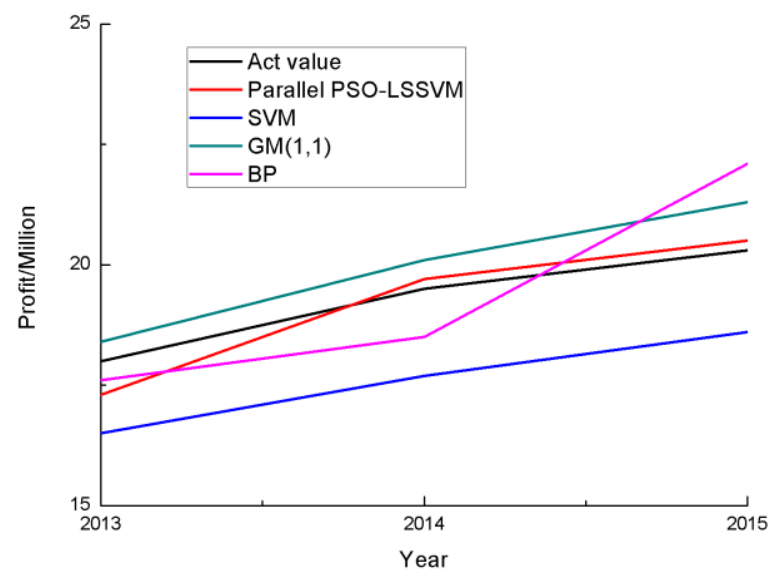

Figure 6. Predictive Effect Comparison Chart 
From fig 6, we can see that the improved parallel PSO-LSSVM method significantly improves the prediction accuracy. Compared with other methods, the prediction accuracy of the improved parallel PSO-LSSVM method is the highest, and it is the closest to the actual value. From the above two experiments, we can prove that the reliability and validity of the improved parallel PSO-LSSVM method.

\section{Conclusion}

The emergence of the cloud computing has brought a new innovation for the development of the e-commerce. It is obvious that the cloud computing promotes the ecommerce. It can find in time the existing problems and manage better the e-commerce to forecast the interests of the e-commerce. This paper uses the improved parallel PSOLSSVM method to predict the electronic commerce and achieves the good results. This paper has the following works. Firstly, this paper introduces the relevant backgrounds. Secondly, this paper introduces the cloud computing concepts. Thirdly, this paper proposes the improved parallel PSO-LSSVM algorithm. Fourthly, this paper uses the improved parallel PSO-LSSVM Algorithm to study the profit of the e-commerce. Finally, the experimental results show that the algorithm has the good reliability and validity.

\section{References}

[1] Thepparit Banditwattanawong, Masawee Masdisornchote, Putchong Uthayopas. Multi-provider cloud computing network infrastructure optimization [J]. Future Generation Computer Systems, 2016, 55: $116-128$

[2] Georg Lackermair. Hybrid cloud architectures for the online commerce [J]. Procedia Computer Science, 2011, 3: 550-555.

[3] Nayer A. Hamidi, G.K. Mahdi Rahimi, Alireza Nafarieh, Ali Hamidi, Bill Robertson. Personalized Security Approaches in E-banking Employing Flask Architecture over Cloud Environment [J]. Procedia Computer Science, 2013, 21:18-24.

[4] Wang Yi. Research and implementation of customer behavior and sales forecast model of electronic commerce [D]. Capital Normal University, information management, 2009.

[5] Ren Jianfeng, Zhang Xinxiang. Research on E-business customer churning modeling and prediction [J]. Computer Simulation, 2012, 5: 363-366.

[6] Zhang Yanrong. Research on Prediction of E-commerce consumer behavior based on rough Set [J]. Theory Commercial Research, 2014, 12: 101-106

[7] Ju Chunhua, Lu Qibei, Guo Pengfei. E-commerce customer churn prediction model combined with individual activity [J]. Systems Engineering-Theory \& Practice, 2013, 1: 141-150.

[8] E-commerce credit risk prediction base on PCA-SVR [J]. Journal of Modern Information, 2015, 35: 7679.

[9] Zi Zhidao. Forecasting and analysis of cross border electronic commerce based on $\mathrm{GM}(1,1)$ [J]. Mathematics in practice and theory, 2015, 1: 97-105.

[10] Nan Hua, Cristian Morosan, Agnes DeFranco. The other side of technology adoption: Examining the relationships between e-commerce expenses and hotel performance [J]. International Journal of Hospitality Management, 2015, 45: 109-120.

[11] Adel. A. Alyoubi. E-commerce in Developing Countries and how to develop them during the Introduction of Modern Systems [J]. Procedia Computer Science, 2015, 65: 479-483.

[12] Sherah Kurnia, Jyoti Choudrie, Rahim Md Mahbubur, Basil Alzougool. E-commerce technology adoption: A Malaysian grocery SME retail sector study [J]. Journal of Business Research, 2015, 68(9): 1906-1918.

[13] Steven S. Aanen, Damir Vandic, Flavius Frasincar. Automated product taxonomy mapping in an ecommerce environment [J]. Expert Systems with Applications, 2015, 42: 1298-1313.

[14] Eleni Kavvadia, Spyros Sagiadinos, Konstantinos Oikonomou, Giorgos Tsioutsiouliklis, Sonia Aïssa. Elastic virtual machine placement in cloud computing network environments [J]. Computer Networks, 2015, 93: 435-447.

[15] Omar Ali, Jeffrey Soar, Jianming Yong. An investigation of the challenges and issues influencing the adoption of cloud computing in Australian regional municipal governments [J]. Journal of Information Security and Applications, In Press, Corrected Proof, Available online 11 December 2015.

[16] Alireza Shameli-Sendi, Makan Pourzandi, Mohamed Fekih-Ahmed, Mohamed Cheriet. Taxonomy of Distributed Denial of Service mitigation approaches for cloud computing [J]. Journal of Network and Computer Applications, 2015, 58: 165-179. 
[17] Ibrahim Alzamil, Karim Djemame, Django Armstrong, Richard Kavanagh. Energy-Aware Profiling for Cloud Computing Environments [J]. Electronic Notes in Theoretical Computer Science, 2015, 318: 91108.

[18] Youming Lin, Teemu Kämäräinen, Mario Di Francesco, Antti Ylä-Jääski. Performance evaluation of remote display access for mobile cloud computing [J]. Computer Communications, 2015, 72: 17-25.

[19] Frank M. Götz. Genolab-Forensic biostatistics with cloud computing [J]. Forensic Science International: Genetics Supplement Series, 2015, 5: e438-e440.

[20] Acklesh Prasad, Peter Green. Governing cloud computing services: Reconsideration of IT governance structures [J]. International Journal of Accounting Information Systems, 2015, 19:45-58.

[21] Hui Lin, Li Xu, Xinyi Huang, Wei Wu, Yijie Huang. A trustworthy access control model for mobile cloud computing based on reputation and mechanism design [J]. Ad Hoc Networks, 2015, 35: 51-64.

[22] Bin Wang, Zhengwei Qi, Ruhui Ma, Haibing Guan, Athanasios V. Vasilakos. A survey on data center networking for cloud computing [J]. Computer Networks, 2015, 91: 528-547.

[23] Shahin Vakilinia, Mustafa Mehmet Ali, Dongyu Qiu. Modeling of the resource allocation in cloud computing centers [J]. Computer Networks, 2015, 91: 453-470. 
International Journal of Grid and Distributed Computing Vol. 9, No. 6 (2016) 